\title{
GEMEINSAME EVALUIERUNG DER INNOVATIONSSCHECK-PROGRAMME
}

\author{
RENATE HANDLER
}

\section{AUSGANGSLAGE}

$\mathrm{D}$ e Innovationsscheck-Programme (I-Scheck-Programme) umfassen zwei Formate, die von der Forschungsförderungsgesellschaft mbH (FFG) im Rahmen eines speziell auf KMU zugeschnittenen Paketes von FTI-Fördermaßnahmen (KMU-Paket) abgewickelt werden:

- Der Innovationsscheck (I-Scheck) wurde im November 2007 als gemeinsame Initiative des vormaligen Bundesministeriums für Wissenschaft, Forschung und Wirtschaft (bmwfw) und des Bundesministeriums für Verkehr, Innovation und Technologie (bmvit) gestartet und bietet eine max. Förderhöhe von € 5.000,ohne Selbstbehalt bei einer Laufzeit von 12 Monaten. Anträge konnten laufend bis zum 31.12.2017 eingereicht werden.

- Der Innovationsscheck-plus (I-Scheck-plus) wurde im Juni 2011 als Initiative des bmwfw gestartet und bietet eine max. Förderhöhe von $€ 10.000$,- bei einem Selbstbehalt von $20 \%$ und einer Laufzeit von 12 Monaten. Anträge konnten laufend bis zum 31.12.2017 eingereicht werden.

Mit einem Budget für die gesamte Programmlaufzeit für beide Formate von 33,2 Mio. Euro (22,2 Mio. Euro I-Scheck, 11,0 Mio. Euro I-Scheck-plus) handelt es sich damit, im Vergleich zum FFG-Gesamtförderbudget (im Jahr 2015 alleine 343 Mio. Euro Barwert ${ }^{1}$ ), um kleine Förderprogramme

Die I-Scheck-Programme richten sich dabei speziell an kleinere, bisher nicht regelmäßig innovierende Unternehmen, die kein eigenes FgEPersonal haben und daher auf den Wissenstransfer von Forschungseinrichtungen angewiesen sind. In ihrer Interventionslogik verfolgen die Förderprogramme drei Zielcluster, die in Abbildung 1 dargestellt sind.
Begründen lässt sich eine öffentliche Intervention in Form der 1Scheck-Programme damit, dass die Ergebnisse von FEl-Aktivitäten, ähnlich wie Bildung, ein meritorisches Gut darstellen: Unternehmen, die keine FEl betreiben, können den Wert von FEl auch nicht richtig einschätzen und investieren daher zu wenig in solche Leistungen. Bringt man sie aber in Kontakt mit „FEl-ExpertInnen“ und unterstützt die ersten gemeinsamen Projekte, können sie den Mehrwert unmittelbar erfahren, ihre Investitionen entsprechend anpassen und so in nachhaltige FEl-Aktivitäten einsteigen. Die I-Scheck-Programme sind somit ein Instrument, um die Nutzungsbarrieren für das meritorische Gut „Forschung, Entwicklung und Innovation" für KMU zu beseitigen.

\section{METHODISCHE HERANGEHENSWEISE DER EVALUIERUNG}

convelop wurde im Sommer 2016 mit der gemeinsamen Evaluierung beider I-Scheck-Programme beauftragt (Auftraggeber: Bundesministerium für Verkehr, Innovation und Technologie und ehemals Bundesministerium für Wissenschaft, Forschung und Wirtschaft, heute Bundesministerium für Digitalisierung und Wirtschaftsstandort). Die Aufgabenstellung war, die Konzeption und Umsetzung der Programme zu analysieren und zu bewerten, wie weit die Ziele tatsächlich erreicht wurden. Aufgebaut werden konnte auf Evaluierungen der einzelnen I-Scheck-Formate, die bereits 2011 (I-Scheck) und 2015 (I-Scheck plus) durchgeführt wurden. Es sollten Optimierungspotenziale aufgedeckt

\begin{tabular}{|c|c|c|}
\hline \multicolumn{3}{|c|}{ Zielkategorien der Innovationsscheck-Programme } \\
\hline $\begin{array}{l}\text { Verbreitern der FEI-Basis } \\
\text { • Verbreiterung der österreichischen } \\
\text { Forschungs- und Innovationsbasis durch } \\
\text { Heranführen von KMU an regelmäßige } \\
\text { F\&E- bzw. Innovationsleistung }\end{array}$ & $\begin{array}{c}\text { Aktivieren von Wissenstransfer } \\
\text { • Stimulierung des Wissenstransfers } \\
\text { zwischen KMU und Wissenschaftssektor und } \\
\text { Schließung der Wissenslücke } \\
\text { - Abbau der Schwellenangst von KMU } \\
\text { gegenüber den wissenschaftlichen } \\
\text { Forschungseinrichtungen } \\
\text { - Erhöhung der Kooperationsfähigkeit } \\
\text { und -Bereitschaft zwischen KMU und } \\
\text { wissenschaftlichen Forschungseinrichtungen }\end{array}$ & $\begin{array}{c}\text { Forcieren und Verbessern } \\
\text { von FEI-Vorhaben } \\
\text { • KMU setzen FEI-Vorhaben um bzw. können } \\
\text { FEI-Vorhaben effizienter und effektiver } \\
\text { durchführen und rascher zur Marktreife führen }\end{array}$ \\
\hline
\end{tabular}

Abbildung 1: Zielkategorien der Innovationsscheck-Programme 
und Empfehlungen zur Weiterentwicklung der Programme abgeleitet werden.

Um diese Aufgaben zu erfüllen, wurde ein Methoden-Mix eingesetzt. Neben der Analyse relevanter Dokumente hat das Projektteam die FFG-Monitoringdaten (Zeitraum: Programmstart bis 28.6.2016) ausgewertet, sowie vertiefende qualitative Erhebungen bei Unternehmen, Forschungseinrichtungen, Programmmanagement und den involvierten Ministerien durchgeführt (Interviews und Fokusgruppen). Erstmalig erfolgte auch eine Verschneidung der Daten zu den I-Scheck-beziehenden Unternehmen mit der F\&E-Erhebung der Statistik Austria. Die Ergebnisse dieses Analyseschrittes werden in Box 1 zusammenfassend dargestellt.

\section{BOX 1: F\&E-AKTIVITÄTEN DER GEFÖRDERTEN UNTERNEHMEN}

Im Rahmen der Evaluierung wurde eine Verschneidung der Unternehmensdaten der I-Scheck-Beziehenden mit Daten der F\&E-Erhebung der Statistik Austria des Zeitraums 2007-2013 durchgeführt. Ziel dieses Analyseschrittes war es, ein umfassenderes Bild über die FGE-Aktivitäten der I-Scheck-Unternehmen zu erhalten. Da diese Erhebung auf F\&E fokussiert, werden reine Innovationsaktivitäten darin nicht erfasst. Die Fallzahl der Unternehmen, die nur einen I-Scheck-plus erhalten haben, war aufgrund der kurzen Laufzeit des Programmes (ab 2011) gering und wurde in den Detailauswertungen nicht berücksichtigt. Zusammengefasst standen folgende Fragestellungen im Zentrum dieser Sonderauswertung:

- Tauchen I-Scheck-Unternehmen in der F\&E-Erhebung auf: wenn ja, zu welchem Zeitpunkt und wie hoch sind ihre F\&E-Ausgaben?

- Welche Bedeutung haben die I-Scheck-Unternehmen insgesamt für die Gruppe der F\&E-aktiven Unternehmen?

Abbildung 2 gibt einen Überblick über die Ergebnisse dieser Verschneidung.

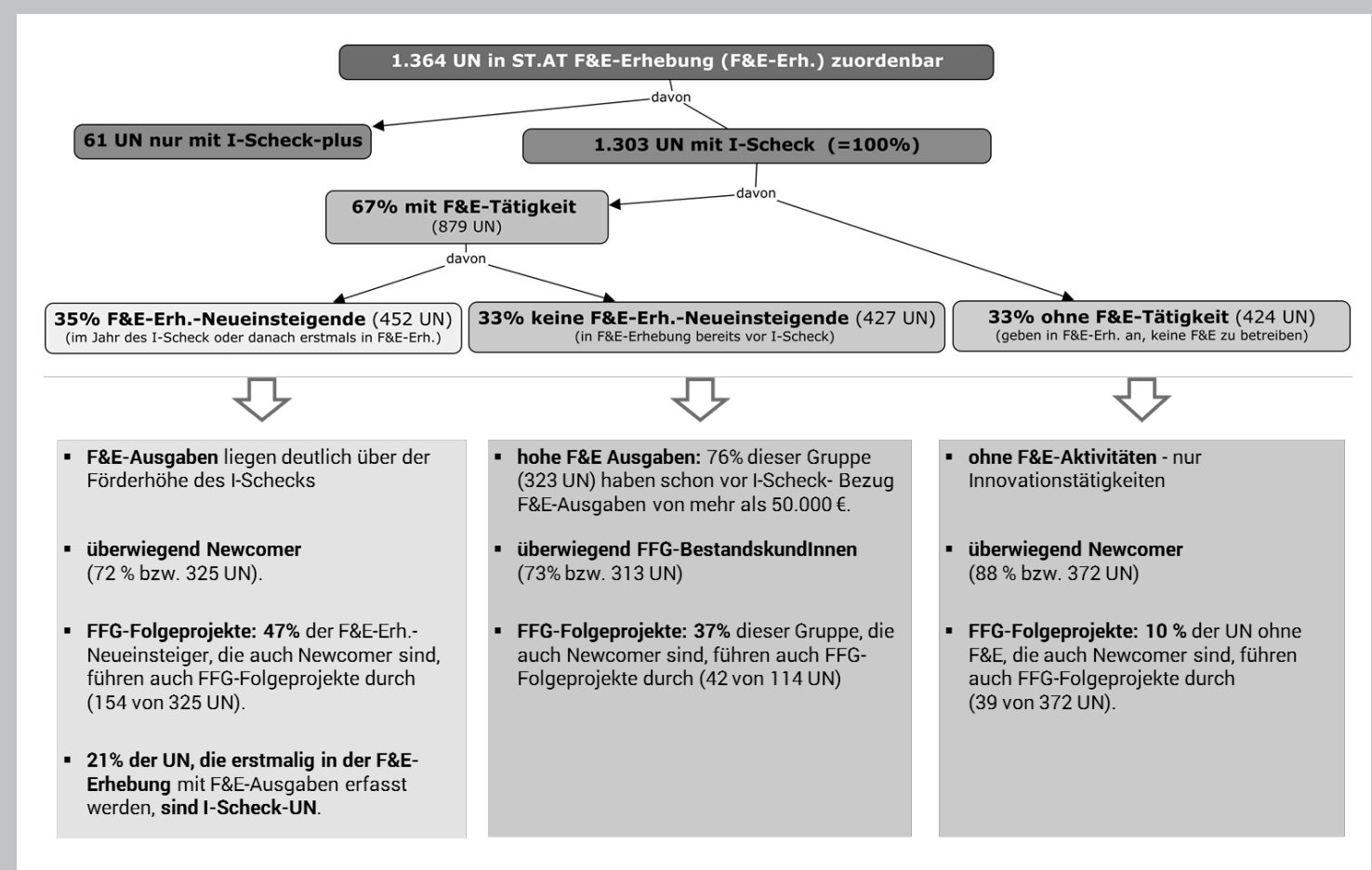

Abbildung 2: Überblick zu I-Scheck-Unternehmen (UN) in der F\&E-Erhebung (2007-2013)

Quelle: Daten: Statistik Austria, Darstellung: convelop

Die Darstellung enthält gerundete Prozentsätze, daher ergibt die Summe nicht immer 100\% (vgl. 35\% FqE-Erhebungs-Neueinsteigende, 33\% keine F\&E-Erhebungs-Neueinsteigende, 33\% ohne F\&E-Tätigkeit). 


\section{DIE PROGRAMMZIELE WERDEN VERFOLGT}

Gemäß der drei Programmzielcluster zeigen die Ergebnisse der Evaluierung, dass die zugehörigen Wirkungslinien verfolgt werden:

\section{VERBREITERUNG DER FEI-BASIS: NEWCOMER WERDEN MOBILISIERT}

Die Auswertungen der FFG-Monitoringdaten und die Verschneidung mit der F\&E-Erhebung der Statistik Austria zeigen, dass mit Hilfe des ISchecks eine große Gruppe von Unternehmen neu für die FEl-Förderung gewonnen werden konnte (68\% der Antragstellenden bei den I-ScheckProgrammen sind Newcomer ${ }^{2}$ ) und dass die I-Schecks auch mit einer Hebelwirkung für die FEl-Ausgaben der Newcomer verbunden waren (die F\&E-Ausgaben der erstmalig in der F\&E-Erhebung erfassten Unternehmen liegen deutlich über der Förderhöhe des I-Schecks).

Rund ein Viertel der Newcomer führte außerdem Folgeprojekte bei der FFG durch und zeigte damit einen nachhaltigen Einstieg in FEl-Aktivitäten. Allerdings wagten sich weniger als die Hälfte dieser Unternehmen an komplexere FEI-Vorhaben heran. Der Rest verließ das Einsteigerniveau der I-Scheck-Programme nicht.

\section{AKTIVIEREN VON WISSENSTRANSFER: NEUE KO- OPERATIONEN MIT VIELFÄLTIGEM MEHRWERT}

Die Auswertungen der FFG-Monitoringdaten haben ergeben, dass im Evaluierungszeitraum (Programmstart bis 28.6.2016) beinahe 4.500 I-Scheck- und I-Scheck-plus-Projekte von über 3.700 Unternehmen umgesetzt wurden. Darüber hinaus konnten Interviews und Fokusgruppen aufzeigen, dass neue nachhaltige Netzwerke zwischen Forschungseinrichtungen und Unternehmen entstanden sind, Forschungseinrichtungen davon profitiert haben, ihr Wissen in der Praxis zu erproben und Unternehmen Innovationen wirtschaftlich verwerten konnten, wie es ohne I-Schecks nicht möglich gewesen wäre. Ergänzt wurden diese Effekte durch vielfältige Impulse, die Unternehmen und Forschungseinrichtungen für ihre laufende Arbeit aus den Kooperationen mitnehmen konnten (Tabelle 1).

\section{FORCIEREN UND VERBESSERN VON FEI-VORHA- BEN: HOHE ADDITIONALITÄT UND VERBESSERUNG DER OUALITÄT}

Interviews und Fokusgruppen lieferten deutliche Belege dafür, dass ohne Unterstützung durch I-Schecks(-plus) FEI-Vorhaben der teilnehmenden KMU zum Teil nicht, oder nur langsamer realisiert worden wären, und dass die in den Projekten erarbeiteten Ergebnisse die Qualität der geplanten Innovationen steigern konnten. Darüber hinaus gibt es eine Reihe weiterer Effekte wie z.B. leichtere Vermarktung neu entwickelter
Produkte durch ein „wissenschaftliches Label”, mehr Bereitschaft der Unternehmen mit neuen Ideen zu experimentieren und "Multiplikatoreffekte" für die Vermarktung neuer Produkte durch die Forschungseinrichtungen mit ihren Publikationen und Konferenzbeiträgen.

\begin{tabular}{|c|c|}
\hline Für Unternehmen & Für Forschungseinrichtungen \\
\hline $\begin{array}{l}\text { + Einstiegsinstrument für erstmalige } \\
\text { Kooperation mit FE, Innovation } \\
\text { gewinnt an Stellenwert in den UN } \\
+ \text { Ergebnisse sind nützlich, um } \\
\text { Entwicklungsvorhaben rascher und } \\
\text { mit höherer Qualität umzusetzen (bis } \\
\text { hin zur Patentanmeldung), Vorhaben } \\
\text { wären ohne Scheck nicht, langsamer } \\
\text { oder anders realisiert worden } \\
+ \text { Perspektivenerweiterung durch Im- } \\
\text { pulse und Erkenntnisse der FE; auch } \\
\text { negative Ergebnisse sind nützlich, } \\
\text { um bestimmte Ideen auszuschließen } \\
+ \text { Teilaspekte von FEI-Vorhaben, für } \\
\text { die keine Ressourcen vorhanden } \\
\text { sind, können ausgelagert werden } \\
+ \text { Neue Ideen können auf ihr Potenzial } \\
\text { getestet werden } \\
+ \text { Strategisches Instrument, um } \\
\text { Kooperationsmöglichkeiten mit FE } \\
\text { zu testen } \\
+ \text { Wissenschaftliche Legitimation für } \\
\text { Marketing wertvoll (Qualitätssiegel, } \\
\text { FE als Multiplikator) }\end{array}$ & $\begin{array}{l}\text { + Instrument zur Erweiterung des } \\
\text { Netzwerks zu UN mit der Aussicht } \\
\text { auf Folgekooperationen } \\
+ \text { Strategisches Instrument, mit dem } \\
\text { UN aktiv akquiriert werden } \\
+ \text { Wissenschaftlicher Nutzen: Impuls } \\
\text { für Publikationen und Konferenz- } \\
\text { beiträge } \\
+ \text { Verbindung von Wissenschaft und } \\
\text { Praxis (Praxistest) } \\
+ \text { Impulse für Themenerweiterung, } \\
\text { neue Perspektiven } \\
+ \text { Einbindung von Studierenden in } \\
\text { Praxisprojekte (über Projektarbeiten, } \\
\text { Abschlussarbeiten) } \\
\text { Übernahme von Studierenden in die } \\
\text { UN kommt vor } \\
+ \text { Praxisbeispiele für die Lehre } \\
\text { - Als Drittmittel untergeordnet, } \\
\text { größere Projekte haben Vorrang }\end{array}$ \\
\hline
\end{tabular}

Tabelle 1: Nutzen der I-Scheck-Programme für die Unternehmen (UN) bzw. Forschungseinrichtung (FE)

Quelle: Interviews und Fokusgruppen, Zusammenstellung convelop

\section{I-SCHECK-PROGRAMME ERREICHEN SPEZIFISCHE ZIELGRUPPEN}

Die I-Scheck-Programme sind auf kleine, bisher nicht regelmäßig innovierende Unternehmen ausgerichtet, die sie überwiegend auch tatsächlich erreichen. So sind 68\% der I-Scheck(-plus)-Unternehmen Newcomer $^{2}$ bei der FEl-Förderung.

Vertiefende Ergebnisse zur Bewertung der Zielgruppenerreichung konnten im Rahmen der Evaluierung insbesondere die erstmalig erfolgte Verschneidung der erfassten Unternehmen im FFG-Monitoring mit der F\&E-Erhebung der Statistik Austria (Zeitraum 2007-2013) liefern: Mindestens $21 \%$ aller neu in der F\&E-Erhebung der Statistik Austria erfassten Unternehmen scheinen in dieser Erhebung auf, nachdem sie einen I-Scheck-(plus) bezogen haben. 
Neben den FEl-Neulingen erreichen die Programme aber auch FEIerfahrene Unternehmen. Für diese entsteht ein substanzieller Mehrwert aus den I-Scheck-Projekten, der zwar dem Geist der Programme entspricht, in den Programmzielen aber nicht explizit reflektiert wird (z.B. Experimente mit neuen Ideen und neuen Kooperationsbeziehungen, die sonst nicht durchgeführt worden wären).

\section{HERAUSFORDERNDER ÜBERGANG ZU KOMPLEXEREN FÖRDERPROGRAMMEN}

Die I-Scheck-Programme sind Teil des KMU-Paketes der FFG, in dessen Rahmen sie als Einstiegsinstrumente fungieren. Wie die Auswertung der FFG-Monitoringdaten zeigt, sind sie tatsächlich ein gutes Instrument um ins FFG-Fördersystem einzusteigen. Dass Unternehmen in Folge auch komplexere Förderprogramme in Anspruch nehmen, findet allerdings nur in beschränktem Ausmaß (10\% der Newcomer) statt.

Interviews und Fokusgruppen zeigen, dass der Sprung von den ISchecks zur Feasability Study, zum Basisprogramm oder zu noch komplexeren FFG-Programmen nicht leicht zu bewältigen ist. Begründet wird dies damit, dass der administrative Aufwand für die Beantragung und die qualitativen Anforderungen an die Projekte zu hoch und der geforderte Selbstbehalt zu umfassend sind.

Dieses Ergebnis ist jedoch nicht auf das Design der I-Scheck-Programme zurückzuführen. Vielmehr deuten die Befunde auf das Fehlen ähnlich niederschwelliger Programme hin, mit denen FEl-Aktivitäten der FEl-Einsteigenden fortgesetzt werden könnten.

\section{EMPFEHLUNGEN AUF BASIS DER EVALUIERUNGSERGEBNISSE}

Wie die Evaluierung gezeigt hat, werden die Ziele der I-Scheck-Programme verfolgt, die Zielgruppen werden erreicht und darüber hinaus werden nicht unmittelbar intendierte positive Effekte erzeugt. Die I-Scheck-Programme kommen somit ihrer Funktion nach und beseitigen Nutzungsbarrieren für das meritorische Gut „Forschung, Entwicklung und Innovation“ für KMU. Zudem sind die I-Scheck-Programme gut in die FTI-Strategie des Bundes „Der Weg zum Innovation Leader" im Bereich „Potenziale der Innovation aktivieren" eingebettet und adressieren deren Ziele.

Basierend auf diesen Evaluierungsergebnissen wird eine Fortführung der I-Scheck-Programme angesichts ihres bisherigen Erfolges und ihres Beitrages zu den Zielen der FTI-Strategie empfohlen. Grundsätzlich sind beide Formate positiv zu bewerten. Es gibt allerdings auch Hinweise auf Anpassungsbedarf, aus denen sich zwei Szenarien für die Weiterführung ableiten lassen:

\section{SZENARIO A: BEIBEHALTEN VON ZWEI SCHECKFOR- MATEN MIT ANPASSUNGEN}

In diesem Szenario werden beide Schecks beibehalten, gleichzeitig wird aber auch deren Fördersumme valorisiert. Beim I-Scheck sollte die
Fördersumme erhöht werden, weil sich nach Einschätzung der InterviewpartnerInnen, der auch die Evaluierenden folgen, mit € 5.000,- sinnvolle FqE-Projekte kaum noch finanzieren lassen. Beim I-Scheck-plus wäre eine Anpassung der Fördersumme als Folge auf die Erhöhung beim I-Scheck nötig, um auch weiterhin eine sinnvolle Differenzierung zwischen den Schecks zu ermöglichen.

\section{SZENARIO B: STREICHEN DES I-SCHECKS UND BEIBE- HALTEN DES I-SCHECK-PLUS}

Die rückläufigen Antragszahlen beim I-Scheck zeigen, dass eine FEIFörderung mit nur $€ 5.000$,- zunehmend an Attraktivität verliert, auch wenn eine selbstbehaltsfreie Förderung ein wirkungsvolles Instrument darstellt, um Nutzungsbarrieren von meritorischen Gütern zu beseitigen (z.B. Cold-Call-Akquisition durch FE). Der I-Scheck würde in diesem Szenario daher nicht verlängert.

Weiterzuführen wäre das bisherige Format des I-Scheck-plus, sowohl in der Höhe der Fördersumme von bis zu € 10.000,-- als auch mit einem Selbstbehalt. Die Fördersumme reicht aus, um kleinere FEl-Vorhaben anzustoßen, und der Selbstbehalt signalisiert eine Identifikation der Unternehmen mit den geförderten Projekten. Allerdings sollte in der Kommunikation mit der Zielgruppe verstärkt darauf hingewiesen werden, dass die Fördersumme des I-Schecks nicht zur Gänze ausgeschöpft werden muss und damit die absolute Höhe des Selbstbehalts bei kleineren Projekten aliquot verringert werden kann

\section{8: NEUE PROGRAMMLINIE „INNOVATIONSSCHECK MIT SELBSTBEHALT“}

Im Zuge der Weiterentwicklung der I-Scheck-Programme sind die Ergebnisse dieser Evaluierung in ein neues Format eingeflossen, das dem dargestellten Szenario B des Evaluierungsteams folgt: Mit dem „Innovationsscheck mit Selbstbehalt" steht seit 2. Jänner 2018 eine gemeinsam getragene Programmlinie des Bundesministeriums für Digitalisierung und Wirtschaftsstandort und des Bundesministeriums für Verkehr, Innovation und Technologie zur Verfügung. Die Sonderrichtlinien, die den bisherigen I-Scheck-Programmen (Innovationsscheck und Innovationsscheckplus) zugrunde liegen, haben per 31.12.2017 ihre Gültigkeit verloren.

Evaluierungsteam convelop: Thomas Jud, Renate Handler, Stephan Kupsa, Simon Pohn-Weidinger

\section{AUTORIN}

\section{RENATE HANDLER}

convelop - cooperative knowledge design gmbh

E: renate.handler@convelop.at 